\title{
THREE-DIMENSIONAL GRAVITY-MAGNETIC CROSS-GRADIENT JOINT INVERSION BASED ON STRUCTURAL COUPLING AND A FAST GRADIENT METHOD*
}

\author{
Yuanping Zhang and Yanfei Wang ${ }^{1)}$ \\ Key Laboratory of Petroleum Resources Research, Institute of Geology and Geophysics, \\ Chinese Academy of Sciences, Beijing 100029, China; \\ University of Chinese Academy of Sciences, Beijing 100049, China; \\ Innovation Academy for Earth Science, Chinese Academy of Sciences, Beijing 100029, China \\ E-mail: yfwang@mail.iggcas.ac.cn, zhangyuanpinghaida@163.com
}

\begin{abstract}
In order to effectively solve the low precision problem of the single gravity density inversion and the magnetic susceptibility inversion, and the limitation of the gravity-magnetic joint inversion method based on the petrophysical parameter constraint, this paper studies the three-dimensional gravity-magnetic cross-gradient joint inversion based on the structural coupling and the fast optimization algorithm. Based on the forward and inversion modeling of three-dimensional gravity density and three-dimensional magnetic susceptibility using the same underground grid, along with cross-gradient coupling as the structural constraint, we propose a new gravity-magnetic joint inversion objective function including the data fitting term, the total variation regularization constraint term and the crossgradient term induced by the structural coupling. The depth weighted constraint and the data weighting constraint are included into the objective function, which requires different physical property models to minimize their respective data residuals. At the same time, the cross-gradient term tends to zero, so that the structure of the gravity and magnetic models tends to be consistent. In realization, we address a fast and efficient gradient algorithm to iteratively solve the objective function. We apply this new joint inversion algorithm to the 3D gravity-magnetic model inversion test and compare it with the results of a single inversion algorithm. The experimental tests of synthetic data indicate that the gravity-magnetic cross-gradient joint inversion method can effectively improve the accuracy of the anomaly position and numerical accuracy of the inverted anomaly physical parameters compared with the single physical inversion method.
\end{abstract}

Mathematics subject classification: 86-08, 65J20, 65K10.

Key words: Joint inversion, Gravity, Magnet, Cross-gradient, Regularization.

\section{Introduction}

Gravimetric and magnetic explorations are two effective methods in geophysical exploration. Both methods are volume exploration and can be performed quickly and economically. The gravimetric method is widely used to dig out about the deep structure of the earth, to divide the structural units, and to detect minerals. The magnetic method also plays an important role in many aspects, a typical application is to find non-ferrous metal minerals. In the gravimetric and magnetic exploration, it is necessary to invert the distribution of the underground anomalies

\footnotetext{
* Received October 29, 2018 / Revised version received March 28, 2019 / Accepted May 5, 2019 /

Published online October 24, 2019 /

1) Corresponding author.
} 
according to the data to guide the follow-up work reasonably and effectively. Advances in the gravimetric and magnetic techniques play a crucial role in the exploration technology, as the inversion results can indicate important information such as the distribution of subsurface density (or susceptibility) and the shape of the model. However, the geophysical exploration targets which we are facing are much more complicated and difficult than before (such as complex surface conditions and underground structures), making it difficult to obtain high-quality data from geophysical methods such as gravity strategies [31]. In addition, different geophysical exploration methods have their own properties for the imaging of media underground. Especially for the gravimetric and magnetic exploration, the most important problem for the inversion of the field data is the inherent ill-posedness [19,20]. There are many reasons for the ill-posedness of the inverse problem, including: different anomalous bodies can correspond to the same observation data, insufficient observation data, environmental interference and observation errors caused by limited instrument accuracy $[19,20]$. To reduce the ill-posedness of the inverse problem and improve the accuracy of inversion results, joint inversion method become a tendency in geophysics $[7,8,12,14,23]$. Joint inversion methods, which comprehensively using the information obtained by various exploration methods, can effectively depict the underground geological structure.

The joint inversion strategy between different physical parameters can be divided into two categories. One is the coupling method of petrophysical parameters, which is a joint inversion by establishing an empirical relationship between two or more physical parameters [18]. For example, based on the empirical relationship between density and velocity, Jegen et al. in [18] developed a joint inversion method for gravity, magnetism, and seismology. However, in most cases, the empirical relationship between these physical parameters depends on the specific geological conditions; therefore, the limitations of this kind of method are relatively strong. The other type of method belongs to the model structure coupling method, which performs joint inversion by finding the structural consistency of two or more physical models $[6,15,22,26,27]$. Among the joint inversion methods, the representative method is the crossgradient joint inversion method based on the structural coupling proposed by Gallardo and Meju in $[7,8]$. This method achieved satisfactory results in the joint inversion of seismic travel time and direct current (DC) resistivity method. This approach is to make their structures tend to be consistent by the cross-gradient values between the models tending to zero. This method neither depends on the empirical relationship between physical parameters, nor on specific geological conditions, and has received extensive attention [4,10,12,14,23,25,35]. For example, Moorkamp et al. in [23] proposed a joint inversion framework for three-dimensional MT, gravity and seismic refraction data based on cross-gradients; Abubakar et al. in [1] combined crossgradient method with petrophysical method to realize surface wave and magnetotelluric joint inversion; Fregoso et al. in [5] combined Euler deconvolution with cross-gradient and applied it to gravity-magnetic joint inversion, which achieved good results; Gao et al. in [13] developed an efficient 3D seismic travel time and DC resistivity joint inversion strategy based on the cross-gradient structure constraint, their synthetic model tests showed that the joint inversion imaging results improve the resolution of the solution compared with single physical property imaging.

In order to effectively solve the problem of the low precision of the single physical property imaging method, e.g., the gravity inversion or the magnetic susceptibility inversion, and the limitation of the gravity-magnetic joint inversion method based on the petrophysical parameter constraint, in this paper, a three-dimensional gravity-magnetic cross-gradient joint inversion 
strategy based on the structural coupling theory is studied. Based on the forward and inversion of the three-dimensional gravity density and three-dimensional magnetic susceptibility, and the united mesh grid underground, together with the cross-gradient coupling as the structural constraint, we propose a new gravity-magnetic joint inversion scheme, which includes the data fitting term, the total variation regularization constraint term and the cross-gradient term. To locate the anomaly position correctly, the depth weighted constraint and the data weighting constraint are added to the objective function, which requires different physical parameter models to minimize their residuals of the simulated data to the measurement data. Meanwhile, as the cross-gradient term tends to zero, the structure of the gravity and magnetic models tends to be consistent. In solving methodology, we apply an efficient gradient descent algorithm to iteratively solve the minimization of the objective function. We give comparison of the twodimensional and three-dimensional joint inversion of gravity-magnetic models and the single inversion scheme. The experimental tests indicate that the gravity-magnetic cross-gradient joint inversion method can effectively improve the accuracy of the depth position and numerical precision of the inverted anomaly comparing with the single physical inversion method.

\section{Forward and Inverse Problems of Single Gravity and Magnetism}

\subsection{Forward and inverse problems of gravity}

According to the law of universal gravitation, we calculate the gravity anomaly which generated by geological bodies beneath the ground at a certain point on the surface or in the air. First, we calculate the gravitational potential generated by the residual mass of the geological body at the measuring point, and then we calculate the gravity anomaly which is the first derivative of the gravitational potential along the direction of gravity. We create a Cartesian coordinate system and we take a point $\mathrm{O}$ on the ground as the coordinate origin, the $\mathrm{Z}$ axis is vertically downward, and $\mathrm{X}$ axis and $\mathrm{Y}$ axis compose the horizontal plane. We assume that the residual density of the abnormal body is $\sigma$, and the volume element in the abnormal body is $d v=d \xi d \eta d \zeta$ which has a coordinate $(\xi, \eta, \zeta)$, then the residual mass of every volume element is $d m=\sigma d \xi d \eta d \zeta$. Now, we need to calculate the gravity anomaly at the point A with coordinates $(\mathrm{x}, \mathrm{y}, \mathrm{z})$, and the distance from the residual mass $d m$ to point $\mathrm{A}$ is

$$
r=\left[(\xi-x)^{2}+(\eta-y)^{2}+(\zeta-z)^{2}\right]^{1 / 2},
$$

and the gravitational potential $V(x, y, z)$ generated by the residual mass of the abnormal body and measured at the point $\mathrm{A}$ is:

$$
V(x, y, z)=\lambda \iiint_{v} \frac{\sigma d \xi d \eta d \zeta}{\left[(\xi-x)^{2}+(\eta-y)^{2}+(\zeta-z)^{2}\right]^{1 / 2}},
$$

where $\lambda$ is the gravitational parameter.

Since the $\mathrm{Z}$ axis direction is consistent with the direction of gravity, the first derivative of the gravitational potential of the residual mass along the $\mathrm{Z}$ direction is the gravity anomaly:

$$
\begin{aligned}
\Delta g & =\frac{\partial V}{\partial z}=V_{z}=\lambda \iiint_{v} \frac{\sigma(\zeta-z) d \xi d \eta d \zeta}{\left[(\xi-x)^{2}+(\eta-y)^{2}+(\zeta-z)^{2}\right]^{3 / 2}} \\
& =\lambda \iiint_{v} \frac{\sigma(\zeta-z)}{r^{3}} d \xi d \eta d \zeta .
\end{aligned}
$$


The formulation (2.2) can be written in simplified form as a compact form:

$$
d_{1}=G_{1} m_{1}
$$

where $G_{1}$ is the integral operator maps the parameter $m_{1}$ from model space to observation space, the kernel function can be written as

$$
G_{1}=\lambda \frac{\zeta-Z}{\left[(\xi-x)^{2}+(\eta-y)^{2}+(\zeta-z)^{2}\right]^{3 / 2}}=\lambda \frac{\zeta-Z}{r^{3}},
$$

for simplicity of notation, we still use $G_{1}$ to represent the kernel function, $d_{1}$ is the gravity anomaly and the parameter $m_{1}$ is the residual density.

For the single gravity density inversion problem, we calculate the residual density value by minimizing the residual between the observed data and the theoretical value of the gravity anomaly. However, a principal difficulty with the inversion of gravity data (also for magnetic data) is the inherent nonuniqueness that exists in any geophysical method based upon a static potential field. Meanwhile, the gravity and magnetic methods have a relatively high resolution in the lateral direction, but its longitudinal resolution is weak. To overcome these difficulties, we have to introduce prior information into the inversion so that a unique solution is obtained [20].

Generally, we need to solve a least-squares problem. However, due to the ill-posedness of the gravity (and magnetic) inversion problem, the calculation result is unstable [28-30]. To solve this problem, we add regularization constraints in the objective function to reduce the ill-posedness during the calculation. There are two main types of regularization constraints used in the calculation of the potential field inversion. One is the smooth constraint regularization defined by the smooth norm, such as the $L_{2}$ norm; the other is non-smooth constrained regularization defined by non-smooth norm such as $L_{1}$ norm, total variation (TV) norm, and so forth. Both types of regularization constraints have their own advantages and disadvantages.

If the inversion object is smooth, the Tikhonov smooth constrained regularization model defined by $L_{2}$ norm can be established. The objective function is defined as:

$$
\varphi_{g}^{\text {Tikh }}=1 / 2\left\|W_{d}^{1 / 2} \cdot\left(G_{1} \cdot m_{1}-d_{1}^{\mathrm{obs}}\right)\right\|_{2}^{2}+{ }^{\alpha_{1} / 2}\left\|W_{z} \cdot m_{1}\right\|_{2}^{2},
$$

where $d_{1}^{\text {obs }}$ is the observed gravemetric field anomaly, $\alpha_{1}>0$ is the regularization parameter, $W_{d}$ and $W_{z}$ are the data and model weighting matrices, respectively; meanwhile, $W_{z}$ is only related with depth $z$.

The other formulation is the Tikhonov non-smooth constrained regularization model defined by non-smooth norm. In the gravity inversion problem, the model is often piecewise constant or piecewise continuous, and non-smooth regularization constraints have advantages for dealing with this type of problem and the boundary of the model can be depicted more clearly. The TV norm model is used here, and the objective function is defined as:

$$
\varphi_{g}^{\mathrm{TV}}=1 / 2\left\|W_{d}^{1 / 2} \cdot\left(G_{1} \cdot m_{1}-d_{1}^{\mathrm{obs}}\right)\right\|_{2}^{2}+\tilde{\alpha}_{1} \int_{V}\left|\nabla m_{1}(r)\right| d v,
$$

where $\nabla$ is the differential operator, $\tilde{\alpha}_{1}>0$ is the regularization parameter. Considering the model weighting factor, the formulation (2.5) is further changed to the following form

$$
\varphi_{g}={ }^{1 / 2}\left\|W_{d}^{1 / 2} \cdot\left(G_{1} \cdot m_{1}-d_{1}^{\mathrm{obs}}\right)\right\|_{2}^{2}+{ }^{\alpha_{1} / 2}\left\|W_{z} \cdot m_{1}\right\|_{2}^{2}+\tilde{\alpha}_{1} \int_{V}\left|\nabla m_{1}(r)\right| d v .
$$


In $(2.6)$, the TV stabilizing functional can be approximated by $w_{\mathrm{sTV}}(r)=\sqrt{\left|m_{1}(\mathrm{r})\right|^{2}+\varsigma^{2}}$, where $\varsigma$ is a small constant number.

Total variation norm used in this formulation aims to keep edge structure of the anomaly. The total variation term tries to punish oscillation on the boundary by "the norm of the gradient". The parameter $\varsigma$ is set to be 0.001 during calculations. Instead of keeping edge structure, if we consider "sparsity" of anomalies, the Huber function which yields the "Huber norm" [30], can be considered as a hybrid $l_{1}-l_{2}$ error measure that is robust to outliers. Therefore, in consideration of the "sparsity", we believe that the Huber function may be a surrogate of the TV term for proper values of the parameter $\varsigma$, since it is good in dealing with "outliers", and it will be our next stage of study.

Considering the fact that the potential field function decays rapidly with increasing of the depth, the inversion results tend to be concentrated near the surface; this may lead to the low vertical resolution and the inaccurate inversion results. Here, the depth weighting function proposed by Li et al. in [19] is added to offset the effect of the fast decay of the potential field function as the depth increases. The depth weighting function is defined as:

$$
w(z)=\frac{1}{\left(z+z_{0}\right)^{\beta / 2}},
$$

where $z$ is the buried depth of the center point of the block unit, $z_{0}$ and $\beta$ are constants.

Meanwhile, we consider that the potential field function also has the characteristic of rapid attenuation with the increase of horizontal relative distance. In order to improve the stability of the calculation, we add the data weighting function to the data fitting term of the objective function [24]. The data weighting function is defined as:

$$
W_{d}^{1 / 2}=\operatorname{diag}\left(\sqrt{\sum_{i}\left(G_{j i}^{-1}\right)^{2}}\right) .
$$

To perform a gravity inversion, two steps are required: (1) we first mesh the model area and carry out the forward simulation calculation of gravity anomaly; (2) we develop some efficient optimization methods for solving the minimization problem.

\subsection{Forward and inverse problems of magnetism}

In our research, we assume that the effects of remanence and demagnetization are ignored, only the homogeneous uniform magnetization phenomenon is considered. According to the basic theory of the magnetic field, the relationship between the magnetization $M$ and the external magnetic field strength $H$ can be expressed as:

$$
M=\kappa H,
$$

where $H$ represents the background magnetic field and $\kappa$ is the magnetic susceptibility.

According to the relationship between the magnetic field strength and the magnetic induction, the relationship between the magnetic potential $U\left(r_{p}\right)$ and the magnetic susceptibility $\kappa$ can be expressed as the form of the first type of Fredholm integral equation:

$$
U\left(r_{p}\right)=\frac{1}{4 \pi} \int_{v} \kappa \cdot H \cdot \nabla_{Q}\left(\frac{1}{r_{P}-r_{Q}}\right) d v=\frac{1}{4 \pi} \int_{v} \kappa \cdot H \cdot \nabla_{Q} \frac{1}{r} d v
$$


where $v$ is the target area with a magnetic anomaly, $r_{P}$ and $r_{Q}$ represent the position of the subsurface and observation point, respectively. We create the same coordinate system as is in the gravity calculation before. The volume element in the abnormal body is $d v=d \xi d \eta d \zeta$ which has a coordinate $(\xi, \eta, \zeta)$, and the observation point has a coordinate (x,y,z). And in (2.10), $r$ is defined the same meaning as before, i.e.,

$$
r=r_{P}-r_{Q}=\left[(\xi-x)^{2}+(\eta-y)^{2}+(\zeta-z)^{2}\right]^{1 / 2}
$$

According to the magnetic potential formula (2.10), the magnetic anomaly of the geological body underground is the first derivative of $U\left(r_{P}\right)$. And it can be further expressed in the operator form

$$
d_{2}=G_{2} m_{2}
$$

where $d_{2}$ is the magnetic field anomaly, $G_{2}$ is the integral operator acts as a mapping from parameter (model) space to the observation space, again as in the gravimetric forward formulation, the operator $G_{2}$ is characterized by a kernel function and $m_{2}$ is the magnetic susceptibility. For simplicity of notation, we still denote the kernel function as $G_{2}$ which is expressed in the form:

$$
G_{2}=G_{x} \cos I \cos A+G_{y} \cos I \sin A+G_{z} \sin I,
$$

and the functions $G_{x}, G_{y}$ and $G_{z}$ have the following forms, respectively:

$$
\begin{aligned}
G_{x} & =\frac{\mu_{0}}{4 \pi} \int_{\nu} \frac{1}{r^{5}}\left\{H_{x}\left[2(x-\xi)^{2}-(y-\eta)^{2}-(z-\zeta)^{2}\right]+H_{y} 3(x-\xi)(y-\eta)+H_{z} 3(x-\xi)(z-\zeta)\right\} d \nu, \\
G_{y} & =\frac{\mu_{0}}{4 \pi} \int_{\nu} \frac{1}{r^{5}}\left\{H_{y}\left[2(y-\eta)^{2}-(x-\xi)^{2}-(z-\zeta)^{2}\right]+H_{x} 3(x-\xi)(y-\eta)+H_{z} 3(y-\eta)(z-\zeta)\right\} d \nu, \\
G_{z} & =\frac{\mu_{0}}{4 \pi} \int_{\nu} \frac{1}{r^{5}}\left\{H_{z}\left[2(z-\zeta)^{2}-(x-\xi)^{2}-(y-\eta)^{2}\right]+H_{x} 3(x-\xi)(z-\zeta)+H_{y} 3(y-\eta)(z-\zeta)\right\} d \nu .
\end{aligned}
$$

In which, $\mu_{0}$ is the vacuum permeability, $H_{x}, H_{y}$, and $H_{z}$ represent the components of the Earth's background magnetic field strength in the three directions of $x, y$ and $z$, respectively. In (2.12), the parameter $I$ represents the magnetic dip and $A$ represents the magnetic declination.

Considering the individual magnetic susceptibility inversion problem, similar to the gravity inversion, two inversion models can be established. One is the Tikhonov smooth constrained regularization model defined by $L_{2}$ norm:

$$
\varphi_{m}^{\text {Tikh }}={ }^{1} / 2\left\|W_{d}^{1 / 2} \cdot\left(G_{2} \cdot m_{2}-d_{2}^{\text {obs }}\right)\right\|_{2}^{2}+{ }^{\alpha_{2} / 2}\left\|W_{z} \cdot m_{2}\right\|_{2}^{2}
$$

where $\alpha_{2}>0$ is the regularization parameter, $W_{d}$ and $W_{z}$ are the data and model weighting matrices, respectively.

For the magnetic inversion problem, considering irregular shape of the anomaly, the nonsmooth constrained regularization model can be applied. The TV-norm constrained model is again used here. The objective function is defined as:

$$
\varphi_{m}^{\mathrm{TV}}={ }^{1} / 2\left\|W_{d}^{1 / 2} \cdot\left(G_{2} \cdot m_{2}-d_{2}^{\mathrm{obs}}\right)\right\|_{2}^{2}+\tilde{\alpha}_{2} \int_{V}\left|\nabla m_{2}(r)\right| d v
$$

where $d_{2}^{\text {obs }}$ is the observed magnetic field anomaly, $\tilde{\alpha}_{2}>0$ is the regularization parameter and $\nabla$ is the differential operator. Considering the model weighting factor, the above formulation 
is changed as the following form

$$
\varphi_{m}={ }^{1 / 2}\left\|W_{d}^{1 / 2} \cdot\left(G_{2} \cdot m_{2}-d_{2}^{\mathrm{obs}}\right)\right\|_{2}^{2}+{ }^{\alpha_{2} / 2}\left\|W_{z} \cdot m_{2}\right\|_{2}^{2}+\tilde{\alpha}_{1} \int_{V}\left|\nabla m_{2}(r)\right| d v .
$$

Similarly, in (2.16), the TV stabilizing functional can be approximated by

$$
w_{\varsigma \mathrm{TV}}(r)=\sqrt{\left|m_{2}(\mathrm{r})\right|^{2}+\varsigma^{2}},
$$

where $\varsigma$ is a small constant number.

To perform a magnetic inversion, two steps are required similarly as the gravity inversion mentioned before: (1) we first mesh the target area and carry out the forward simulation of the magnetic field anomaly; (2) we use the same efficient optimization methods as that in the gravity inversion for finding an optimal solution.

\section{Joint Inversion of Gravity and Magnetism Based on Cross-gradient Constraint}

\subsection{Cross-gradient formulation}

The cross-gradient function was first proposed by [3] and was subsequently widely used in magnetotelluric data, DC data and seismic data, and gravity-magnetic joint inversion $[4,7-9$, $11,14,16,17,21,23]$.

The three-dimensional cross gradient function is defined as:

$$
\vec{t}\left(m_{1}, m_{2}\right):=\vec{t}(x, y, z)=\nabla m_{1}(x, y, z) \times \nabla m_{2}(x, y, z) .
$$

In the above formula, $\nabla$ is the gradient operator, $m_{1}$ and $m_{2}$ represent density and susceptibility parameters involved in the gravity-magnetic joint inversion, respectively. The cross-gradients criterion requires the problem to satisfy the condition $\vec{t}(m)=0\left(m=\left(m_{1}, m_{2}\right)\right)$, where any spatial changes occurring in both density and susceptibility must point in the same or opposite direction irrespective of the amplitude. In a geological sense, this implies that if a boundary exists, then it must be sensed by both methods in a common orientation regardless of the amplitude of the physical property changes [6]. Taking the three-dimensional case as an example, the $\vec{t}$ can be expanded in three directions:

$$
\begin{aligned}
& t_{x}=\frac{\partial m_{1}}{\partial y} \cdot \frac{\partial m_{2}}{\partial z}-\frac{\partial m_{1}}{\partial z} \cdot \frac{\partial m_{2}}{\partial y} \\
& t_{y}=\frac{\partial m_{1}}{\partial z} \cdot \frac{\partial m_{2}}{\partial x}-\frac{\partial m_{1}}{\partial x} \cdot \frac{\partial m_{2}}{\partial z} \\
& t_{z}=\frac{\partial m_{1}}{\partial x} \cdot \frac{\partial m_{2}}{\partial y}-\frac{\partial m_{1}}{\partial y} \cdot \frac{\partial m_{2}}{\partial x} .
\end{aligned}
$$

In the case of a two-dimensional model, the partial derivative of the model with respect to $\mathrm{y}$-axis direction vanishes, and the two-dimensional cross-gradient function is defined as:

$$
\vec{t}\left(m_{1}, m_{2}\right):=\vec{t}(x, y)=\nabla m_{1}(x, y) \times \nabla m_{2}(x, y)
$$

In this situation, the expansion of $\vec{t}$ has only $t_{y}$ remained.

The definition of the above cross-gradient function is a continuous form. In the practical inversion calculation, it needs to be discretized. In this paper, we use the finite difference 


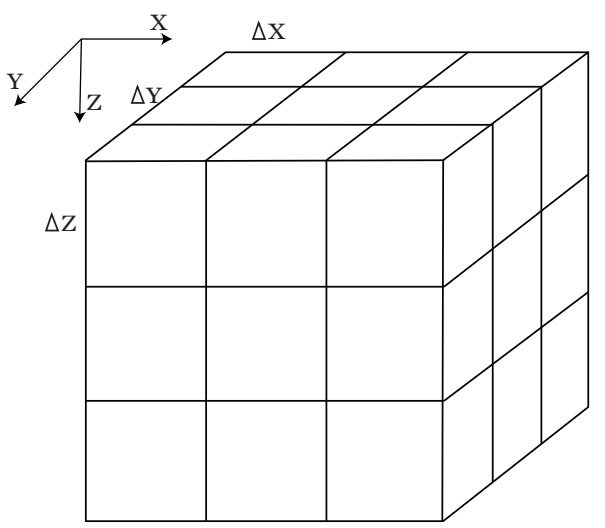

Fig. 3.1. Three-dimensional space meshing diagram underground.

method to discretize the cross-gradient function based on a seven-point central stencil. For the 3D subsurface grid, as shown in Fig. 3.1, the discrete form is as follows:

$$
\begin{array}{r}
t_{x}=\frac{m_{1}(i, j+1, k)-m_{1}(i, j-1, k)}{2 \Delta y} \times \frac{m_{2}(i, j, k+1)-m_{2}(i, j, k-1)}{2 \Delta z} \\
\quad-\frac{m_{1}(i, j, k+1)-m_{1}(i, j, k-1)}{2 \Delta z} \times \frac{m_{2}(i, j+1, k)-m_{2}(i, j-1, k)}{2 \Delta z}, \\
t_{y}=\frac{m_{1}(i, j, k+1)-m_{1}(i, j, k-1)}{2 \Delta z} \times \frac{m_{2}(i+1, j, k)-m_{2}(i-1, j, k)}{2 \Delta x} \\
\quad-\frac{m_{1}(i+1, j, k)-m_{1}(i-1, j, k)}{2 \Delta x} \times \frac{m_{2}(i, j, k+1)-m_{2}(i, j, k-1)}{2 \Delta z}, \\
t_{z}=\frac{m_{1}(i+1, j, k)-m_{1}(i-1, j, k)}{2 \Delta x} \times \frac{m_{2}(i, j+1, k)-m_{2}(i, j-1, k)}{2 \Delta y} \\
\quad-\frac{m_{1}(i, j+1, k)-m_{1}(i, j-1, k)}{2 \Delta y} \times \frac{m_{2}(i+1, j, k)-m_{2}(i-1, j, k)}{2 \Delta x} .
\end{array}
$$

The gradient and cross-product operations are involved in the cross-gradient function, and it has the following properties:

(1) The gradient of a point in the scalar field points to the fastest growing direction, and the magnitude of the gradient is equal to the rate of change of the scalar field at this point;

(2) The cross-product of two vectors is equal to the product of the two vector modules and multiplied by $\sin \theta$, where $\theta$ is the angle between the two vectors. If the two vectors are parallel, therefore the angle is equal to 0 or 180 degrees, and $\sin \theta$ is equal to zero, at which time the cross-product of the two vectors is equal to zero.

Taking the above properties into this geophysical joint inversion, and there are:

(1) When the two physical parameters which participate in the joint inversion change in the same direction, or one of the physical parameters is unchanged, the value of cross-gradient function is zero;

(2) When the gradient of the two physical parameters are not parallel, the cross-gradient function is not equal to zero.

The above properties are the basis of the joint inversion of geophysics based on cross-gradient function. The cross-gradient function is used to connect different physical parameters through 
the structure constraint. Then, we solve the inverse problem by minimizing the objective function with the structural consistency constraint which is defined by the cross-gradient function.

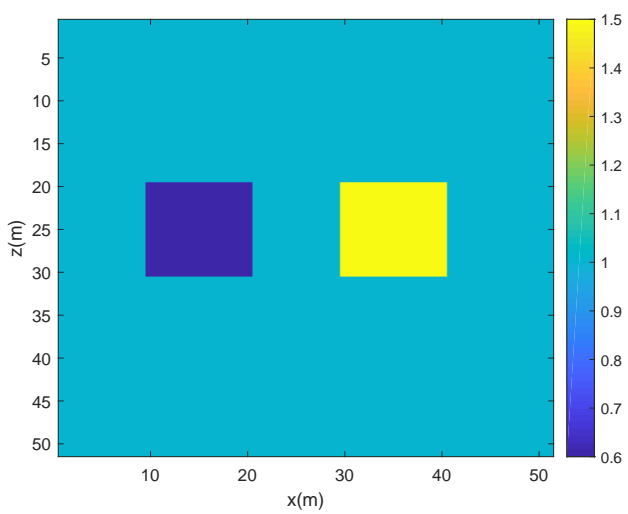

Fig. 3.2. First model consists of two anomalies.

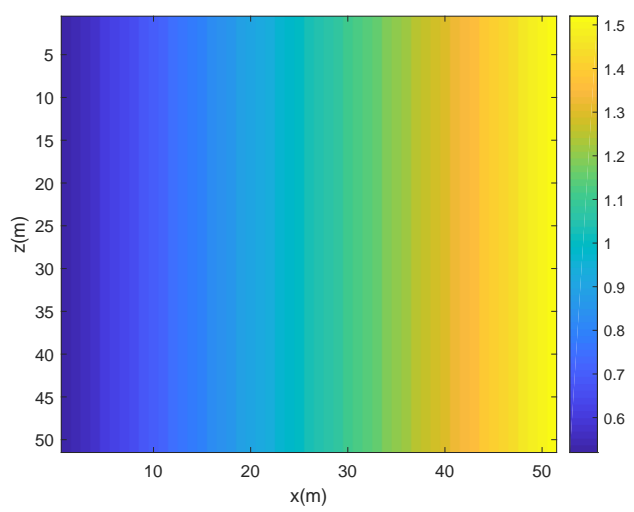

Fig. 3.3. Second model with horizontal changes in $\mathrm{X}$ direction.

In this paper, we use synthetic models to verify the properties of the cross-gradient function. Figs. 3.2 and 3.3 depict the models 1 and 2, respectively. In model 1, two block anomalies, a negative anomaly on the left and a positive anomaly on the right, are constructed. In model 2 , the model values increase laterally from left to right. Fig. 3.4 shows the results of crossgradient between the two models 1 and 2. It can be clearly seen from the Fig. 3.4 that the cross-gradient function values of the two block anomalies are non-zero at the horizontal boundary of the anomalous body, and the other parts are zero. This result means that when the structures are consistent, the cross-gradient value is zero, and when they are not consistent, the value is non-zero. It verifies that the two cross-gradient function properties described before are meaningful. The property of the cross-gradient lays the foundation for using it to the joint inversion, which requires that the cross-gradient value of the density and susceptibility tend to zero during the inversion. Joint inversion based on cross-gradient requires different models to be consistent in their structure and fit the corresponding data as well. 


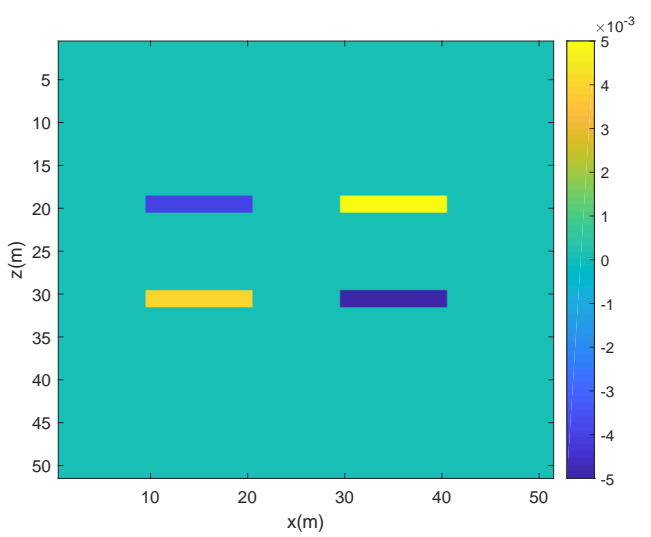

Fig. 3.4. Cross-gradients of the two models in Figs. 3.2 and 3.3.

\subsection{Regularizing joint inversion modeling based on cross-gradient constraint}

The objective function in the gravity-magnetic joint inversion based on the total variation regularization constraint and the cross-gradient constraint is expressed as:

$$
\varphi=\tilde{\varphi}_{g}+\tilde{\varphi}_{m}+\beta \varphi_{\mathrm{cross}}
$$

where $\tilde{\varphi}_{g}$ and $\tilde{\varphi}_{m}$ are approximations to the original functions $\varphi_{g}$ and $\varphi_{m}$, respectively. In equation (3.5):

$$
\begin{aligned}
& \tilde{\varphi}_{g}={ }^{1} / 2\left\|W_{d}^{1 / 2} \cdot\left(G_{1} \cdot m_{1}-d_{1}^{\mathrm{obs}}\right)\right\|_{2}^{2}+{ }^{\alpha_{1} / 2}\left\|W_{z} \cdot m_{1}\right\|_{2}^{2}+\tilde{\alpha}_{1} \sum_{i=1}^{N_{m 1}} \sqrt{\left|\left(D_{1} m_{1}\right)_{i}\right|^{2}+\varsigma^{2}}, \\
& \tilde{\varphi}_{m}=1 / 2\left\|W_{d}^{1 / 2} \cdot\left(G_{2} \cdot m_{2}-d_{2}^{\mathrm{obs}}\right)\right\|_{2}^{2}+{ }^{\alpha_{2} / 2}\left\|W_{z} \cdot m_{2}\right\|_{2}^{2}+\tilde{\alpha}_{2} \sum_{i=1}^{N_{m 2}} \sqrt{\left|\left(D_{2} m_{2}\right)_{i}\right|^{2}+\varsigma^{2}}, \\
& \varphi_{\text {cross }}={ }^{1 / 2}\left\|t\left(m_{1}, m_{2}\right)\right\|_{2}^{2},
\end{aligned}
$$

wherein, the subscript 1 in the above formulae represents gravity data, and the subscript 2 represents magnetic data; $\tilde{\varphi}_{g}$ is the inversion objective function of gravity data, $\tilde{\varphi}_{m}$ is the inversion objective function of magnetic data, $\varphi_{\text {cross }}$ is the cross gradient term, $t=\left[t_{x}, t_{y}, t_{z}\right]^{T}$, $t_{i}(i=x, y, z)$ is in the discrete form given in (3.4), $\alpha_{1}, \alpha_{2}, \tilde{\alpha}_{1}, \tilde{\alpha}_{2}$ are the regularization parameters, $G_{1}$ is the matrix derived from the gravity kernel function, $m_{1}$ is the density value to be calculated, $d_{1}^{\text {obs }}$ is the observed gravity anomaly, $G_{2}$ is the matrix derived from the magnetic kernel function, $m_{2}$ is the magnetic susceptibility value to be calculated, $d_{2}^{\text {obs }}$ is the observed magnetic field anomaly, $\nabla m_{i}$ can be calculated by $D_{i} m_{i}$ with $D_{i}$ the differential operator $(i=1,2)$, $\varsigma$ is a small constant, $W_{d}$ is the data weighting matrix, and $W_{z}$ is the depth weighting matrix.

Linearize the cross-gradient function and perform the first-order Taylor series expansion on 
the three components, ignoring the second-order or higher terms, getting:

$$
\begin{aligned}
& t_{x}\left(m_{1}, m_{2}\right) \approx t_{x}\left(m_{10}, m_{20}\right)+\left[B_{1 x}, B_{2 x}\right]\left[\begin{array}{l}
m_{1}-m_{10} \\
m_{2}-m_{20}
\end{array}\right], \\
& t_{y}\left(m_{1}, m_{2}\right) \approx t_{y}\left(m_{10}, m_{20}\right)+\left[B_{1 y}, B_{2 y}\right]\left[\begin{array}{l}
m_{1}-m_{10} \\
m_{2}-m_{20}
\end{array}\right], \\
& t_{z}\left(m_{1}, m_{2}\right) \approx t_{z}\left(m_{10}, m_{20}\right)+\left[B_{1 z}, B_{2 z}\right]\left[\begin{array}{l}
m_{1}-m_{10} \\
m_{2}-m_{20}
\end{array}\right],
\end{aligned}
$$

, where $t\left(m_{10}, m_{20}\right)$ is the cross gradient between the reference models $m_{10}$ and $m_{20}$.

The partial derivatives of the three cross-gradient components for each model parameter are:

$$
\begin{aligned}
B_{1 x} & =\frac{\partial t_{x}\left(m_{1}, m_{2}\right)}{\partial m_{1}}, & B_{2 x} & =\frac{\partial t_{x}\left(m_{1}, m_{2}\right)}{\partial m_{2}}, \\
B_{1 y} & =\frac{\partial t_{y}\left(m_{1}, m_{2}\right)}{\partial m_{1}}, & B_{2 y} & =\frac{\partial t_{y}\left(m_{1}, m_{2}\right)}{\partial m_{2}}, \\
B_{1 z} & =\frac{\partial t_{z}\left(m_{1}, m_{2}\right)}{\partial m_{1}}, & B_{2 z} & =\frac{\partial t_{z}\left(m_{1}, m_{2}\right)}{\partial m_{2}} .
\end{aligned}
$$

These cross-gradient components can be formulated as a matrix:

$$
B=\left[\begin{array}{ll}
B_{1 x} & B_{2 x} \\
B_{1 y} & B_{2 y} \\
B_{1 z} & B_{2 z}
\end{array}\right]=\left[\begin{array}{ll}
B_{1} & B_{2}
\end{array}\right] .
$$

The gradient values at the density parameter $m_{1}$ and susceptibility parameter $m_{2}$ for the objective function can be written as:

$$
\begin{aligned}
& \frac{\partial \varphi}{\partial m_{1}}=G_{1}^{*} \cdot W_{d} \cdot\left(G_{1} \cdot m_{1}-d_{1}^{o b s}\right)+\alpha_{1} W_{z}^{T} W_{z} m_{1}+\tilde{\alpha}_{1} \cdot L_{1}\left(m_{1}\right) \cdot m_{1}+\beta B_{1} \cdot t, \\
& \frac{\partial \varphi}{\partial m_{2}}=G_{2}^{*} \cdot W_{d} \cdot\left(G_{2} \cdot m_{2}-d_{2}^{o b s}\right)+\alpha_{2} W_{z}^{T} W_{z} m_{2}+\tilde{\alpha}_{2} \cdot L_{2}\left(m_{2}\right) \cdot m_{2}+\beta B_{2} \cdot t,
\end{aligned}
$$

where $G_{i}^{*}$ is the adjoint form of the operator $G_{i}(i=1,2)$. Among them:

$$
\begin{aligned}
& L_{i}(m)=W_{z}^{T} D^{*} \Phi\left(m_{i}\right) D W_{z}, \\
& D=\left[\begin{array}{l}
D_{x} \\
D_{y} \\
D_{z}
\end{array}\right], \quad \mathrm{A}=\operatorname{diag}\left(\frac{1}{\sqrt{\left|\left(D W_{z} m\right)_{i}\right|^{2}+\varsigma^{2}}}\right), \quad \Phi\left(m_{i}\right)=\left[\begin{array}{lll}
A & & \\
& A & \\
& & A
\end{array}\right] .
\end{aligned}
$$

\subsection{An efficient gradient descent method}

The two models $m_{1}$ and $m_{2}$ should be solved iteratively. If we set the values of the $m_{1}$ and $m_{2}$ in (3.12) and (3.13) as constants in advance, e.g., the former iterative points, then the whole iterative processes reduce to solving a series of linear equations. However, this may be time-consuming for large scale computation, e.g., the three-dimensional inversion.

The simplest and the easiest gradient method to program is the steepest descent method

$$
m_{k+1}=m_{k}+\omega_{k} s_{k},
$$


where $m=\left(m_{1}, m_{2}\right), s_{k}=-\nabla \varphi_{k}, \nabla \varphi_{k}=\nabla \varphi\left(m_{k}\right), \omega_{k}$ is the steplength which can be obtained by line search, i.e., optimal $\omega_{k}^{\mathrm{SD}}$ satisfies $\omega_{k}^{\mathrm{SD}}=\operatorname{argmin}_{\omega} \varphi\left(m_{k}+\omega s_{k}\right)$. However, the steepest descent method is slow in convergence and zigzagging after several iterations [32]. Usually in calculations, the line search technique is based on the Armijo-Goldstein line search, which requires the step-length $\omega_{k}$ satisfying [32]

$$
\begin{aligned}
& \varphi\left(m_{k}\right)-\varphi\left(m_{k}+\omega_{k} s_{k}\right) \geq-b_{1} \tau_{k} s_{k}^{T} \nabla \varphi_{k}, \\
& \varphi\left(m_{k}\right)-\varphi\left(m_{k}+\omega_{k} s_{k}\right)<-b_{2} \tau_{k} s_{k}^{T} \nabla \varphi_{k},
\end{aligned}
$$

where $b_{1}<b_{2}$ are two positive parameters. Typical values of $b_{1}$ and $b_{2}$ in calculations are that $b_{1}=0.4$ and $b_{2}=0.9$.

A lot of research works are developed in literature to improve the performance the gradient descent method $[2,34]$. In this paper, we consider the fast monotone gradient descent method in the calculation. This method was proposed by Yuan in [33] for solving the unconstrained optimization problem. The iteration formula is an extension of the steepest descent method except that the stepsize is controlled by

$$
\omega_{k}^{\mathrm{Y}}=\frac{2}{\sqrt{\left(1 / \omega_{k-1}^{\mathrm{SD}}-1 / \omega_{k}^{\mathrm{SD}}\right)^{2}+4\left\|\nabla \varphi_{k}\right\|_{2}^{2} /\left\|s_{k-1}\right\|_{2}^{2}}+1 / \omega_{k-1}^{\mathrm{SD}}+1 / \omega_{k}^{\mathrm{SD}}},
$$

where $s_{k-1}=m_{k}-m_{k-1}$.

Dai and Yuan in [3] proposed a more efficient monotone gradient method based on modification of the formula (3.15):

$$
\omega_{k}^{\mathrm{DY}}=\frac{2}{\sqrt{\left(1 / \omega_{k-1}^{\mathrm{SD}}-1 / \omega_{k}^{\mathrm{SD}}\right)^{2}+4\left\|\nabla \varphi_{k}\right\|_{2}^{2} /\left(\omega_{k-1}^{\mathrm{SD}}\left\|\nabla \varphi_{k}\right\|_{2}\right)^{2}}+1 / \omega_{k-1}^{\mathrm{SD}}+1 / \omega_{k}^{\mathrm{SD}}}
$$

and the specific iterative process can be expressed as:

$$
\omega_{k}= \begin{cases}\omega_{k}^{\mathrm{SD}}, & \text { if } \bmod (k, 4)=1 \text { or } 2 \\ \omega_{k}^{\mathrm{DY}}, & \text { else. }\end{cases}
$$

In their paper, the method is initially designed for convex quadratic programming problem. Noting that the objective functional $\varphi$ is a composite function, the first two terms can be written as a quadratic form, the third term plays a role of edge regularization which is typically small, therefore, the deviation of the objective functional $\varphi$ from a quadratic functional is small. Hence, we can solve the minimization problem via the above fast monotone gradient descent method. The effectiveness of the method works for problem is illustrated in Fig. 4.9.

The iterative algorithm can be outlined as Algorithm 3.1. The diagram of the separate and joint inversion of the gravity and magnetism is shown in Fig. 3.5. 


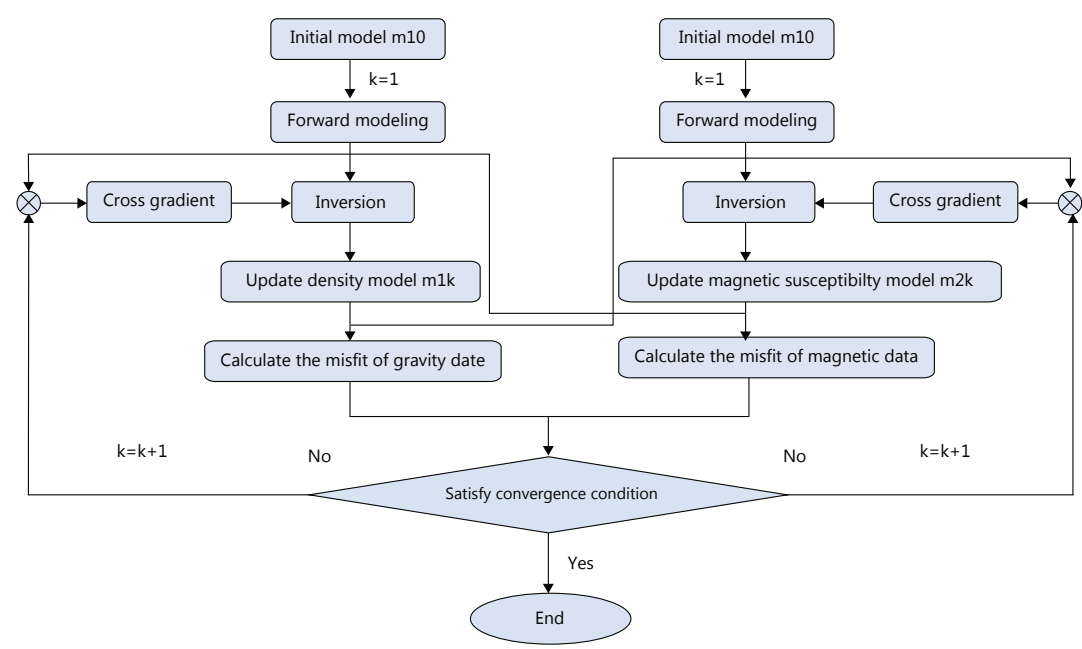

Fig. 3.5. Joint inversion scheme using gravity and magnetism.

Algorithm 3.1. Efficient gradient descent algorithm for joint inversion of gravity and magnetism

Step 1. Input the initial gravity density and magnetic susceptibility models $m_{1}$ and $m_{2}$, and calculate the cross gradient $\vec{t}\left(m_{1}, m_{2}\right)$ between the two models using the formula (3.3);

Step 2. Set the initial value $m_{k}=\left(m_{1}, m_{2}\right)$ as above, calculate the gradient of the objective function $\varphi_{k}$ using formulas (3.10)-(3.13); set $s_{k}=-\nabla \varphi_{k}$; calculate the stepsize $\omega_{k}^{\mathrm{SD}}=\operatorname{argmin}_{\omega} \varphi\left(m_{k}+\omega s_{k}\right)$; set $k:=0$;

Step 3. Update the gravity density $m_{1, k}$ and the magnetic susceptibility $m_{2, k}$ based on the formula (3.14) with $\omega_{k}$ chosen as (3.18);

Step 4. Calculate the residuals between the simulated and the measurement gravity data and the magnetic data, respectively; and determine whether the residuals are small enough. If it is, terminate the iteration and output the solution; If not, set $k:=k+1$ and GOTO Step 2, iterate until the convergence condition is achieved or the maximum number of iterations is reached.

\section{Experimental Tests}

In the numerical experiments, the observation area is set to be a plane area of $260 \mathrm{~m} * 260 \mathrm{~m}$, the data grid is equally spaced, and the grid spacing in the $\mathrm{X}$ and $\mathrm{Y}$ directions is $20 \mathrm{~m}$. We set the underground anomaly area to be a rectangular parallelepiped area of $240 \mathrm{~m} * 240 \mathrm{~m} * 240 \mathrm{~m}$, and divide the area into cubic grid blocks with a side length of $30 \mathrm{~m}$. The distance between the observation plane and the surface is $0 \mathrm{~m}$. In the synthetic test, it is assumed that the magnetic anomaly source is located in a uniform non-magnetic bedrock environment, regardless of the effects of demagnetization and remanence, the magnetic field strength of the background magnetic field is set to $50000 \mathrm{nT}$, and the magnetic dip angle is $90^{\circ}$, and the magnetic declination is $90^{\circ}$.

We remark that in our numerical simulation, we divided the three-dimensional space with 
Table 4.1: Gravity density and magnetic susceptibility of common minerals.

\begin{tabular}{|c|c|c|c|c|c|c|}
\hline Mineral & Magnetite & Hematite & Magnesia & Manganese spinel & Pyrrhotite & Maghemite \\
\hline Density $\mathrm{g} / \mathrm{cm}^{3}$ & $4.9 \sim 5.2$ & $3.4 \sim 4.4$ & $3.6 \sim 4.7$ & $3.57 \sim 3.9$ & $4.3 \sim 4.8$ & 4.88 \\
Susceptibility $\kappa$ & $0.07 \sim 0.2$ & $10^{(-6)} \sim 10^{(-5)}$ & 0.08 & 2.0 & $10^{(-4)} \sim 10^{(-3)}$ & $0.03 \sim 0.2$ \\
\hline
\end{tabular}

equal spacing. The reason we only consider equal-spacing strategy lies in that we do not know where the anomaly exactly located underground. If we can guess the location of the anomaly, the unequal spacing may be applied; accuracy of inversion will be improved. Currently, the equal-spacing strategy has its advantage: it is simple to calculate in practice, especially in the large-scale data calculation problem (note that it is $3 \mathrm{~d}$ in our paper).

In the synthetic test, the constructed anomaly body is a rectangular parallelepiped model with a cross-sectional area of $60 \mathrm{~m} * 60 \mathrm{~m}$, a thickness of $60 \mathrm{~m}$, and the buried depth is $60 \mathrm{~m}$. Its residual density is $5.0 \mathrm{~g} / \mathrm{cm}^{3}$, and the magnetic susceptibility is 0.2 . Table 4.1 lists the gravity density and magnetic susceptibility of some common minerals.
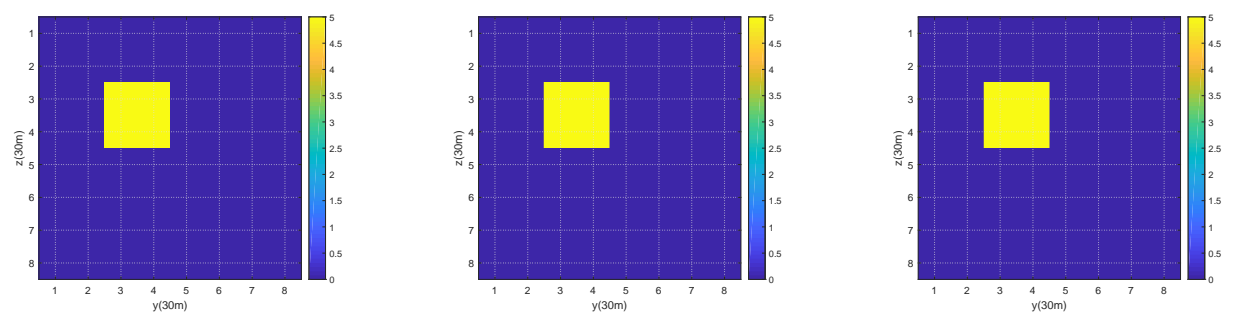

Fig. 4.1. Slices of the true gravity density model along three directions of $\mathrm{X}, \mathrm{Y}$ and Z. (a) $\mathrm{X}=120 \mathrm{~m}$ (b) $\mathrm{Y}=120 \mathrm{~m}(\mathrm{c}) \mathrm{Z}=120 \mathrm{~m}$.
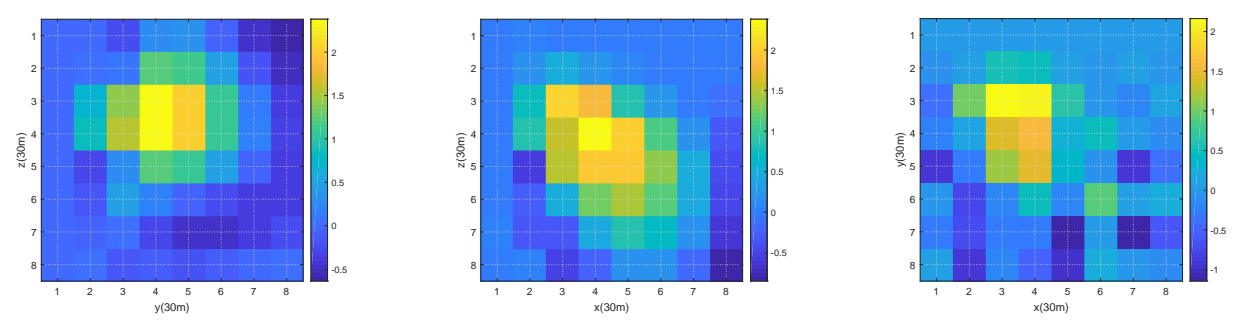

Fig. 4.2. Slices of the gravity density along $\mathrm{X}, \mathrm{Y}$ and $\mathrm{Z}$ directions with separate inversion based on the Tikhonov L2 norm regularization constraint. (a) $\mathrm{X}=120 \mathrm{~m}$ (b) $\mathrm{Y}=120 \mathrm{~m}$ (c) $\mathrm{Z}=120 \mathrm{~m}$.
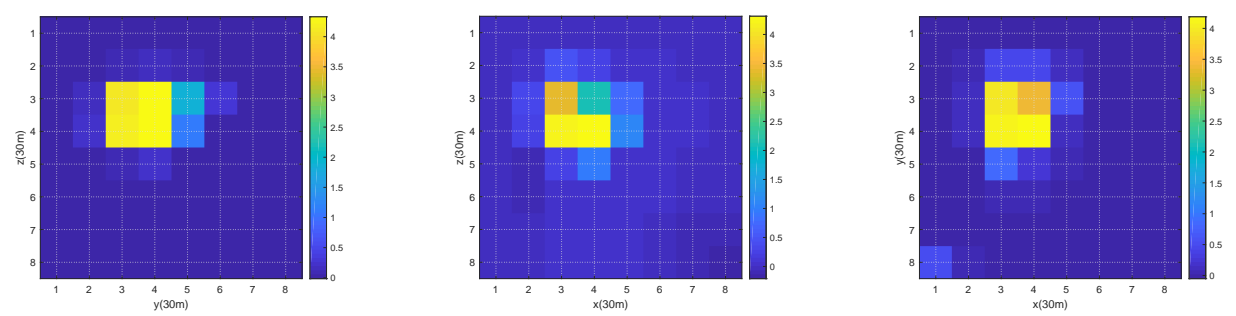

Fig. 4.3. Slices of gravity density along $\mathrm{X}, \mathrm{Y}$ and $\mathrm{Z}$ directions with separate inversion based on the total variation regularization constraint. (a) $\mathrm{X}=120 \mathrm{~m}$ (b) $\mathrm{Y}=120 \mathrm{~m}$ (c) $\mathrm{Z}=120 \mathrm{~m}$. 

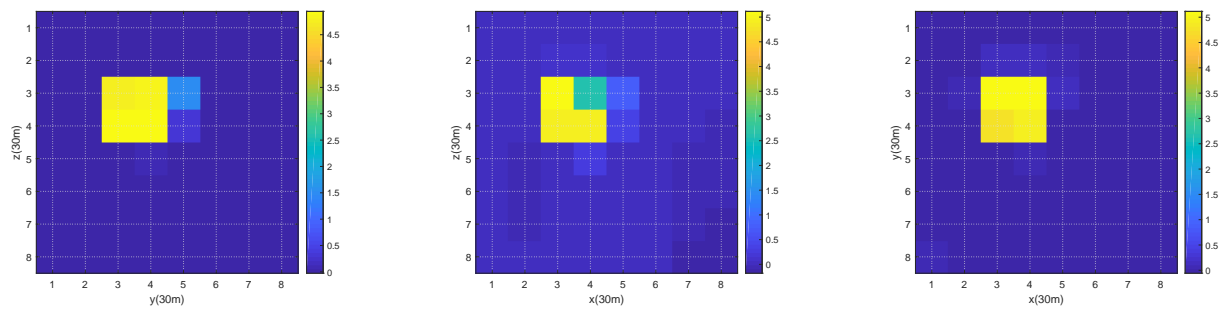

Fig. 4.4. Slices of gravity density along $\mathrm{X}, \mathrm{Y}$ and $\mathrm{Z}$ directions with joint inversion based on the total variation regularization constraint and cross-gradient constraint. (a) X=120m (b) Y=120m (c) $\mathrm{Z}=120 \mathrm{~m}$.
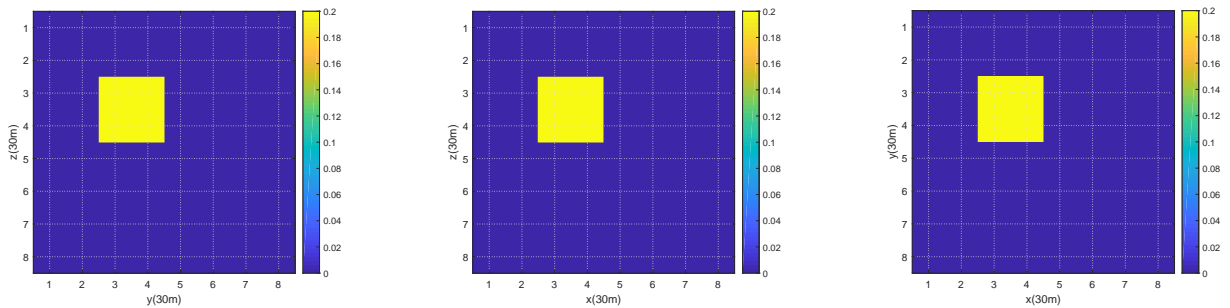

Fig. 4.5. Slices of the true susceptibility model along three directions of $\mathrm{X}, \mathrm{Y}$ and $\mathrm{Z}$. (a) $\mathrm{X}=120 \mathrm{~m}$ (b) $\mathrm{Y}=120 \mathrm{~m}(\mathrm{c}) \mathrm{Z}=120 \mathrm{~m}$.
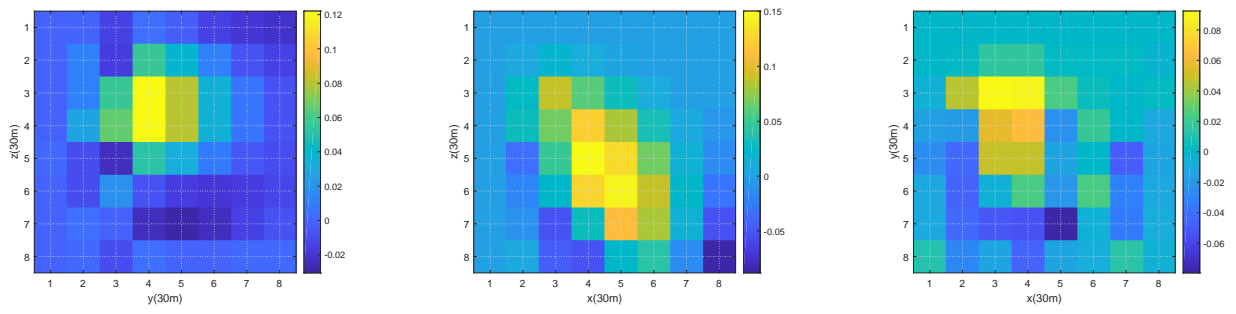

Fig. 4.6. Slices of the magnetic susceptibility along $\mathrm{X}, \mathrm{Y}$ and $\mathrm{Z}$ directions from separate inversion based on the Tikhonov L2 norm regularization constraint. (a) X=120m (b) $Y=120 \mathrm{~m}$ (c) $\mathrm{Z}=120 \mathrm{~m}$.

Fig. 4.1 is a slice diagram of the synthetic three-dimensional gravity density model in the X, $\mathrm{Y}$, and $\mathrm{Z}$ directions, and the residual density of the anomaly body is $5.0 \mathrm{~g} / \mathrm{cm}^{3}$. In all of the Figs. 4.1-4.4, the left-most refers to (a), the middle refers to (b) and the right-most refers to (c). Figs. 4.2 and 4.3 show the inversion results of the density with the separate inversion strategies, i.e., the Tikhonov smooth regularization and the total variation regularization, respectively. Fig. 4.4 gives the results of the inverted density using the joint inversion scheme. Compared with the density model obtained by the separate inversion, the center position of the anomaly is closer to the real model by the joint inversion, and the joint inversion can more accurately describe the model boundary. As a result, the computed model parameter values well approach the standard model residual density values with the joint inversion.

Fig. 4.5 is a slice diagram of the synthetic three-dimensional magnetic susceptibility model in the $\mathrm{X}, \mathrm{Y}$, and $\mathrm{Z}$ directions, and the magnetic susceptibility of the anomaly body is 0.2 . Again, in all of the Figs. 4.5-4.8, the left-most refers to (a), the middle refers to (b) and the right-most refers to (c). Figs. 4.6 and 4.7 show the inversion results of the susceptibility using separate inversion strategies, i.e., the Tikhonov smooth regularization and the total variation 

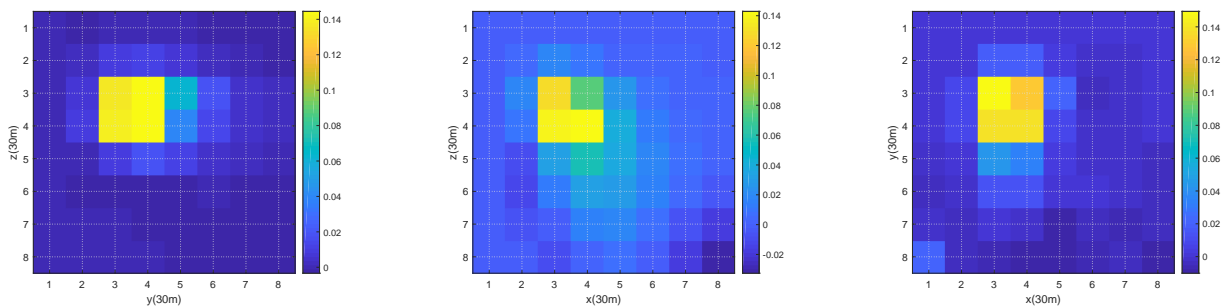

Fig. 4.7. Slices of the true susceptibility model along three directions of X, Y and Z. (a) X=120m (b) $\mathrm{Y}=120 \mathrm{~m}(\mathrm{c}) \mathrm{Z}=120 \mathrm{~m}$.
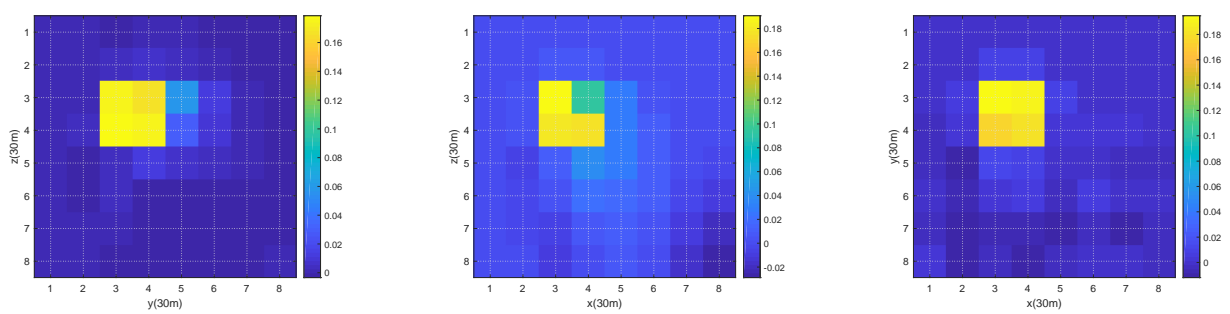

Fig. 4.8. Slices of the magnetic susceptibility along $\mathrm{X}, \mathrm{Y}$ and $\mathrm{Z}$ directions with joint inversion based on the total variation regularization constraint and cross-gradient constraint. (a) $\mathrm{X}=120 \mathrm{~m}$ (b) $\mathrm{Y}=120 \mathrm{~m}$ (c) $\mathrm{Z}=120 \mathrm{~m}$.

regularization, respectively. Fig. 4.8 gives the result of the magnetic susceptibility based on the joint inversion scheme. Compared with the susceptibility model obtained by the separate inversion, the anomaly center position obtained by the joint inversion is closer to the real model, and the joint inversion can more accurately describe the model boundary. As a result, the computed model parameter values are closer to the standard model susceptibility values with the joint inversion.

Fig. 4.9 shows the decreasing curve of the objective functions: the left figure refers to (a), the right refers to (b). It indicates the fast convergence of our algorithm. To show the stability of the inversion algorithm, we apply it to the noisy data, noise level is setting as $5 \%$. The inversion results for gravity data and magnetic data are illustrated in Figs. 4.10 and 4.11,
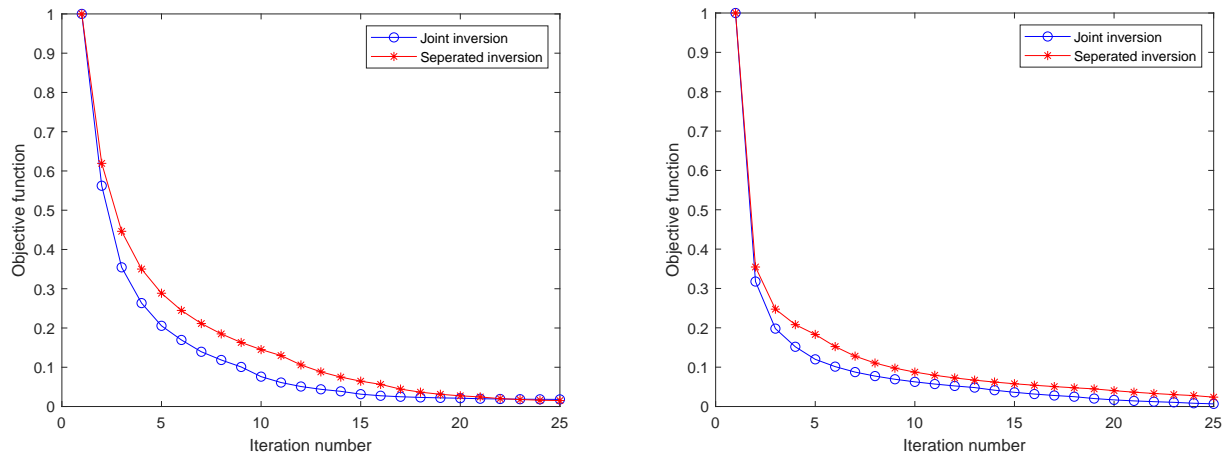

Fig. 4.9. Variation curves of the objective function for gravity density and magnetic susceptibility along with iteration numbers for separate and joint inversions: (a) gravity density data; (b) magnetic susceptibility data. 

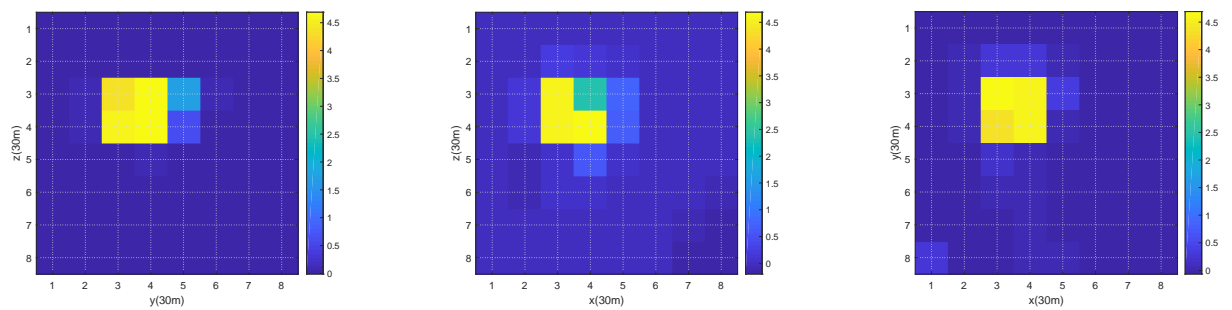

Fig. 4.10. Slices of gravity density along $\mathrm{X}, \mathrm{Y}$ and $\mathrm{Z}$ directions with joint inversion based on total variation regularization constraint and cross-gradient constraint, in which the gravity data contain $5 \%$ random noise. (a) $\mathrm{X}=120 \mathrm{~m}$ (b) $\mathrm{Y}=120 \mathrm{~m}$ (c) $\mathrm{Z}=120 \mathrm{~m}$.
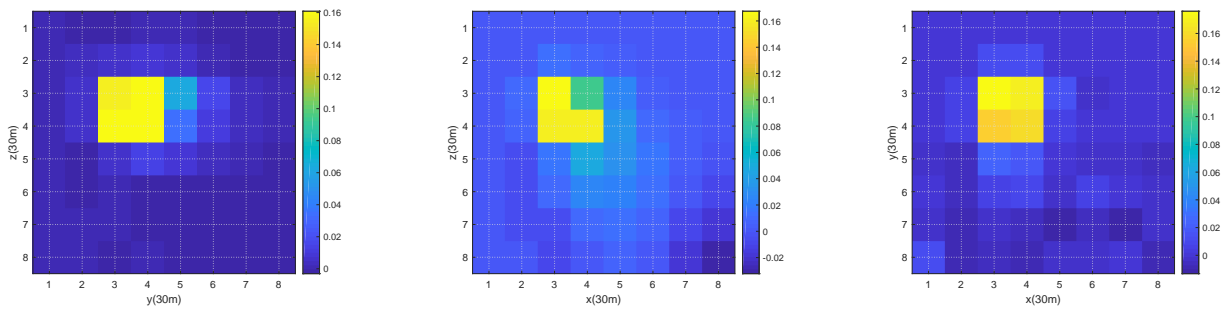

Fig. 4.11. Slices of magnetic susceptibility along $\mathrm{X}, \mathrm{Y}$ and $\mathrm{Z}$ directions with joint inversion based on total variation regularization constraint and cross-gradient constraint, in which the magnetic data contain $5 \%$ random noise. (a) $\mathrm{X}=120 \mathrm{~m}$ (b) $\mathrm{Y}=120 \mathrm{~m}$ (c) $\mathrm{Z}=120 \mathrm{~m}$.

respectively. Similarly, in all of the Figs. 4.10-4.11, the left-most refers to (a), the middle refers to (b) and the right-most refers to (c). It indicates from the two figures that even with noisy data, our algorithm can still generate satisfactory results.

From the perspective of the whole model space, after the joint inversion, the cross-gradients values are relatively small, indicating that the structural constraints using the cross-gradients make the models have better structural consistency, and the inversion results are consistent with the unique characteristics of the geological model structure. Hence the joint inversion strategy improves the rationality of the inversion results.

\section{Discussion and Conclusion}

Improving the reliability of inversion results is a hot topic in the field of geophysical prospecting. Joint inversion technique is an inevitable tendency in geophysical inversion methodology. The emergence of the cross-gradient principle provides a new way of solving the coupling problem of different parameters in the joint inversion. The joint inversion of multiple physical attributes aims to reduce the multiplicity of the model by utilizing various petrophysical properties of the subsurface medium.

In this paper, the gravity-magnetic joint inversion is studied. We mainly focus on the density attribute and magnetic susceptibility attribute of underground anomalies, and consider the cross-gradient method to perform joint inversion experiments. We propose a nonsmooth minimization model based on the cross-constraint and the total variation regularization constraint. To solve the problem, an efficient monotone gradient algorithm is applied. The new inversion scheme with the fast gradient method converges fast to the true solution, and can also balance the data fitting and structural constraints. Based on a three-dimensional gravity density and a magnetic susceptibility model, we tested it using the newly proposed joint inversion process. 
The results show that gravity density inversion imaging and magnetic susceptibility inversion imaging are two effective complementary imaging methods. Gravity density and magnetic susceptibility models can be better restored using joint inversion than the separate inversion strategy.

At the same time, we draw the following conclusions:

(1) The inversion of the gravity/magnetic field is strongly ill-posed problem. The illposedness of the inversion can be effectively reduced by the regularization term and the constraint condition. The inversion results obtained by different regularization terms are also different. The classical Tikhonov smooth regularization method will make the boundary of the inversion result appear excessively smooth. The total variation regularization will be clearer and more accurate for the description of the discontinuous boundary.

(2) Cross-gradient joint inversion, using a variety of physical parameters information, can effectively reduce the ill-posedness of the inversion and improve the quality of the inversion. At the same time, in the joint inversion iteration process, we must pay attention to control the convergence speed of the model. If a model converges faster or slower, the cross gradient of the two models will be too small or too large around the anomaly, which will affect the inverse result.

Acknowledgments. We would like to thank reviewers very much for their valuable comments and suggestions. The research is supported by National Key R \& D Program of the Ministry of Science and Technology of China with the Project "Integration Platform Construction for Joint Inversion and Interpretation of Integrated Geophysics (Grant No. 2018YFC0603500)" and National Natural Science Foundation of China under grant number 91630202.

\section{References}

[1] A. Abubakar, G. Gao, T.M. Habashy and J. Liu, Joint inversion approaches for geophysical electromagnetic and elastic full-waveform data, Inverse Prob., 28:5 (2012), 1-19.

[2] Y.H. Dai, Alternate Step Gradient Method, Optimization, 52:4-5 (2003), 395-415.

[3] Y.H. Dai and Y. Yuan, Analyses of Monotone Gradient Methods, J. Ind. Manag. Optim., 1:2 (2005), 181-192.

[4] E. Fregoso and L.A. Gallardo, Cross-gradients joint 3D inversion with applications to gravity and magnetic data, Geophysics, 74:4 (2009), 31-42.

[5] E. Fregoso, L.A. Gallardo and J. Garcia-Abdeslem, Structural joint inversion coupled with Euler deconvolution of isolated gravity and magnetic anomalies, Geophysics, 80:2 (2015), G67-G79.

[6] L.A. Gallardo and M.A. Meju, Characterization of heterogeneous near-surface materials by joint 2D inversion of dc resistivity and seismic data, Geophys. Res. Lett., 30:13 (2003), 183-196.

[7] L.A. Gallardo, M.A. Perez-flores and E. Gomez-trevino, A versatile algorithm for joint 3D inversion of gravity and magnetic data, Geophysics, 68:3 (2003), 949-959.

[8] L.A. Gallardo and M.A. Meju, Joint two-dimensional DC resistivity and seismic travel time inversion with cross-gradients constraints, J. Geophys. Res.-Sol. Ea., 109:B3 (2004), 1-11.

[9] L.A. Gallardo, M.A. Meju and M.A. Perez-flores, A quadratic programming approach for joint image reconstruction: mathematical and geophysical examples, Inverse Prob., 21:2 (2005), 435452.

[10] L.A. Gallardo, Multiple cross-gradient joint inversion for geospectral imaging, Geophys. Res. Lett., 34:19 (2007), 1-4.

[11] L.A. Gallardo. and M.A. Meju, Joint two-dimensional cross-gradient imaging of magnetotelluric and seismic traveltime data for structural and lithological classification, Geophy. J. R. Astr. Soc., 169:3 (2010), 1261-1272. 
[12] L.A. Gallardo, S.L. Fontes, M.A. Meju, M.P. Buonora and P.P De Lugao, Robust geophysical integration through structure-coupled joint inversion and multispectral fusion of seismic reflection, magnetotelluric, magnetic, and gravity images: example from Santos basin, offshore brazil, Geophysics, 77:5 (2012), 237-251.

[13] J. Gao, H.J. Zhang, H.J. Fang and N. Li, An efficient joint inversion strategy for 3D seismic travel time and DC resistivity data based on cross-gradient structure constraint, Chinese J. Geophys.Ch., 60:9 (2017), 3628-3641. (in Chinese)

[14] J. Gao and H.J. Zhang, An efficient sequential strategy for realizing cross-gradient joint inversion: method and its application to two-dimensional cross borehole seismic travel time and DC resistivity tomography, Geophysical J. Int., 213:2 (2018), 1044-1055.

[15] E. Haber and D. Oldenburg, Joint inversion: a structural approach, Inverse Prob., 13:1 (1997), 63-77.

[16] W.Y. Hu, A. Abubakar and T.M. Habashy, Joint electromagnetic and seismic inversion using structural constraints, Geophysics, 74:74 (2009), 99-109.

[17] V. Infante, L.A. Gallardo, J.C. Montalvo-Arrieta and I.N.D. Len, Lithological classification assisted by the joint inversion of electrical and seismic data at a control site in northeast Mexico, $J$. Appl. Geophys., 70:2 (2010), 93-102.

[18] M.D. Jegen, R.W. Hobbs, P. Tarits and A. Chave, Joint inversion of marine magnetotelluric and gravity data incorporating seismic constraints: preliminary results of sub-basalt imaging off the faroe shelf, Earth Planet. Sc. Lett., 282:1 (2009), 47-55.

[19] Y.G. Li and D.W. Oldenburg, 3-D inversion of magnetic data, Geophysics, 61:2 (1996), 394-408.

[20] Y.G. Li and D.W. Oldenburg, 3-D inversion of gravity data, Geophysics, 63:1 (1998), 109-119.

[21] N. Linde, A. Binley, A. Tryggvason, L.B. Pedersen and A. Revil, Improved hydrogeophysical characterization using joint inversion of cross-hole electrical resistance and ground-penetrating radar traveltime data, Water Resour. Res., 42:42 (2006), 1283-1286.

[22] N. Linde, A. Tryggvason, J.E. Peterson and S.S. Hubbard, Joint inversion of crosshole radar and seismic traveltimes acquired at the south oyster bacterial transport site, Geophysics, 73:73 (2008), G29-G37.

[23] M. Moorkamp, B. Heincke, M. Jegen, A.W. Roberts and R.W. Hobbs, A framework for 3-d joint inversion of MT, gravity and seismic refraction data, Geophysical J. Int., 184:1 (2011), 477-493.

[24] O. Portniaguine and M.S. Zhdanov, 3D magnetic inversion with data compression and image focusing, Geophysics, 67:5 (2000), 1532-1541.

[25] Z. Shi, R.W Hobbs, M. Moorkamp, G. Tian and L. Jiang, 3-D cross-gradient joint inversion of seismic refraction and dc resistivity data., J. Appl. Geophys., 141 (2017), 54-67.

[26] M.D. Stefano, Simultaneous joint inversion for susceptibility and velocity, SEG Technical Program Expanded, (2011), 806-810.

[27] E.M. Syracuse, M. Maceira, G.A. Prieto, H.J. Zhang and C.J. Ammon, Multiple plates subducting beneath Colombia, as illuminated by seismicity and velocity from the joint inversion of seismic and gravity data, Earth Planet. Sc. Lett., 444 (2016), 139-149.

[28] Y.F. Wang and Y.X. Yuan, Convergence and regularity of trust region methods for nonlinear ill-posed inverse problems, Inverse Prob., 21:3 (2005), 821-838.

[29] Y.F. Wang, Computational Methods for Inverse Problems and Their Applications, Higher Education Press (in Chinese), 2007.

[30] Y.F. Wang, Stepanova, Titarenko and Yagola, Inverse Problems in Geophysics and Solution Methods, Higher Education Press (in Chinese), 2011.

[31] P. Yu, J.L. Wang, J.S. Wu and H. Yang, Research Status and Analysis of Geophysical Joint Inversion, Progress Explor. Geophys.-Ch., 29:2 (2006), 87-93. (in Chinese)

[32] Y.X. Yuan, Numerical Methods for Nonlinear Programming, Shanghai: Shanghai Scientific and Technic Publishers, 1993.

[33] Y.X. Yuan, A new stepsize for the steepest descent method, J. Comput. Math., 24:2 (2006), 
149-156.

[34] B. Zhou, L. Gao and Y. H. Dai, Gradient Methods with Adaptive Step-Sizes, Comput. Optim. Appl., 35:1 (2006), 69-86.

[35] J. Zhou, X.H. Meng, L. Guo and S. Zhang, Three-dimensional cross-gradient joint inversion of gravity and normalized magnetic source strength data in the presence of remanent magnetization, J. Appl. Geophys., 119 (2015), 51-60. 